Article

\title{
Properties of Partially Degenerate Complex Appell Polynomials
}

\author{
Dojin Kim ${ }^{\circledR}$ and Sangil Kim * \\ Department of Mathematics, Pusan National University, Busan 46241, Korea; kimdojin@pusan.ac.kr \\ * Correspondence: sangil.kim@pusan.ac.kr
}

Received: 29 October 2019; Accepted: 10 December 2019; Published: 11 December 2019

\begin{abstract}
Degenerate versions of polynomial sequences have been recently studied to obtain useful properties such as symmetric identities by introducing degenerate exponential-type generating functions. As part of our continued work in degenerate versions of generating functions, we subsequently present our study on degenerate complex Appell polynomials by considering a partially degenerate version of the generating functions of ordinary complex Appell polynomials in this paper. We only consider partially degenerate generating functions to retain the crucial properties of the Appell sequence, and we present useful identities and general properties by splitting complex values into their real and imaginary parts; moreover, we provide several explicit examples. Additionally, the differential equations satisfied by degenerate complex Bernoulli and Euler polynomials are derived by the quasi-monomiality principle using Appell-type polynomials.
\end{abstract}

Keywords: Appell polynomials; Complex Appell polynomials; degenerate Appell polynomials; degenerate Bernoulli polynomials; degenerate Euler polynomials

\section{Introduction}

Appell polynomials are one of the largest class of polynomials. The classical Appell polynomials comprise the Bernoulli, Euler, Appell-Hermite, generalized Bernoulli, and generalized Euler polynomials, as well as other examples in [1-4].

The sequence of Appell polynomials $\left\{A_{n}(x)\right\}_{n=0}^{\infty}$ is defined by either of the following identities:

(i)

$$
\begin{gathered}
\frac{d}{d x} A_{n}(x)=n A_{n-1}(x), \quad A_{0}(x) \neq 0, \quad n \in \mathbb{N}, \\
A(t) e^{x t}=\sum_{n=0}^{\infty} A_{n}(x) \frac{t^{n}}{n !}, \quad A(t)=\sum_{n=0}^{\infty} a_{n} \frac{t^{n}}{n !} \quad\left(a_{0} \neq 0\right),
\end{gathered}
$$

where $a_{n}:=A_{n}(0)$ denotes the Appell numbers.

According to the alternative definition in [3], the sequence of Appell polynomials $\left\{A_{n}(x)\right\}_{n=0}^{\infty}$ for $g(t)$ can be equivalently defined by

$$
\frac{1}{g(t)} e^{x t}=\sum_{n=0}^{\infty} A_{n}(x) \frac{t^{n}}{n !}, \quad A_{0}(x) \neq 0
$$

where the generating function satisfies $g(t)=\frac{1}{A(t)}$, and thus (1) is identical to (ii).

Appell polynomials appear in various problems in pure and applied mathematics related to differential equations, approximation theory, interpolation, and summation methods. More examples may be found in $[1,2,5,6]$ (and references therein). Moreover, in theoretical applications, degenerate 
versions of Appell polynomials $\left\{A_{n}(z ; \lambda)\right\}_{n=0}^{\infty}$ of a complex variable $z \in \mathbb{C}$ have been extensively studied [7-11]. These polynomials are defined by the generating functions

$$
\frac{1}{g_{\lambda}(t)} e_{\lambda}^{z}(t)=\sum_{n=0}^{\infty} A_{n}(z ; \lambda) \frac{t^{n}}{n !}, \quad \lambda \in \mathbb{R} \backslash\{0\},
$$

where $g_{\lambda}(t)$ and $e_{\lambda}^{z}(t)$ are obtained from the degenerate exponential functions. That is, the exponential functions $e^{t}$ and $e^{z t}$ are replaced by $e_{\lambda}(t)$ and $e_{\lambda}^{z}(t)$, respectively:

$$
e_{\lambda}(t):=e_{\lambda}^{1}(t):=e^{t_{\lambda}}=(1+\lambda t)^{\frac{1}{\lambda}}, \quad e_{\lambda}^{z}(t):=e^{z t_{\lambda}}=(1+\lambda t)^{\frac{z}{\lambda}}, \quad t_{\lambda}:=\frac{1}{\lambda} \log (1+\lambda t), \quad \lambda \in \mathbb{R} \backslash\{0\} .
$$

Here, it is noted that $\lim _{\lambda \rightarrow 0} e_{\lambda}^{z}(t)=e^{z t}$ and $\lim _{\lambda \rightarrow 0} g_{\lambda}(t)=g(t)$, because $t_{\lambda} \rightarrow t$ as $\lambda \rightarrow 0$. Since Carlitz in $[12,13]$ introduced degenerate formulas for special numbers and polynomials, the degenerate complex Appell polynomials have been extensively studied to find useful properties and identities. Specifically, degenerate Bernoulli and Euler polynomials of complex variables were introduced in [12], whereas degenerate gamma functions and Laplace transforms were introduced in [14], and their modifications in [9]. The study of degenerate versions of known special numbers and polynomials provides several useful identities and related properties. Although the original sequence $\left\{A_{n}(z)\right\}_{n=0}^{\infty}$ satisfies conditions (i) and (ii), the sequence $\left\{A_{n}(z ; \lambda)\right\}_{n=0}^{\infty}$ of degenerate polynomials no longer satisfies these conditions.

To retain the crucial properties of the Appell sequence, we introduce a new type of degenerate Appell polynomials, which are obtained by partially degenerate generating functions. Some examples are provided in Table 1. The aim of this paper is to present degenerate complex Appell polynomials and some of their properties. In particular, using degenerate Appell polynomials, we derive the differential equations satisfied by certain degenerate complex polynomials based on the quasi-monomiality principle.

\begin{tabular}{|c|c|c|}
\hline Degenerate Appell Polynomials & Degenerate Generating Functions & $g_{\lambda}(t)$ \\
\hline Degenerate Euler polynomials & $\frac{2}{e_{\lambda}(t)+1} e^{z t}=\sum_{n=0}^{\infty} E_{n, \lambda}(z) \frac{t^{n}}{n !}$ & $g_{\lambda}(t)=\frac{e_{\lambda}(t)+1}{2}$ \\
\hline Degenerate Bernoulli polynomials & $\frac{t}{e_{\lambda}(t)-1} e^{z t}=\sum_{n=0}^{\infty} B_{n, \lambda}(z) \frac{t^{n}}{n !}$ & $g_{\lambda}(t)=\frac{e_{\lambda}(t)-1}{t}$ \\
\hline $\begin{array}{l}\text { Type } 2 \text { degenerate Euler } \\
\text { polynomials }\end{array}$ & $\frac{2}{e_{\lambda}^{\frac{1}{2}}(t)+e_{\lambda}^{-\frac{1}{2}}(t)} e^{z t}=\sum_{n=0}^{\infty} E_{n, \lambda}^{(2)}(z) \frac{t^{n}}{n !}$ & $g_{\lambda}(t)=\frac{e_{\lambda}^{\frac{1}{2}}(t)+e_{\lambda}^{-\frac{1}{2}}(t)}{2}$ \\
\hline $\begin{array}{l}\text { Degenerate generalized Bernoulli } \\
\text { polynomials }\end{array}$ & $\frac{t^{\alpha}}{\left(e_{\lambda}(t)-1\right)^{\alpha}} e^{z t}=\sum_{n=0}^{\infty} B_{n, \lambda}^{(\alpha)}(z) \frac{t^{n}}{n !}$ & $g_{\lambda}(t)=\frac{\left(e_{\lambda}(t)-1\right)^{\alpha}}{t^{\alpha}}$ \\
\hline $\begin{array}{l}\text { Degenerate generalized Euler } \\
\text { polynomials }\end{array}$ & $\frac{2^{\alpha}}{\left(e_{\lambda}(t)+1\right)^{\alpha}} e^{z t}=\sum_{n=0}^{\infty} E_{n, \lambda}^{(\alpha)}(z) \frac{t^{n}}{n !}$ & $g_{\lambda}(t)=\frac{\left(e_{\lambda}(t)+1\right)^{\alpha}}{2^{\alpha}}$ \\
\hline $\begin{array}{l}\text { Degenerate generalized Bernoulli } \\
\text { polynomials of order } m\end{array}$ & $\begin{array}{c}\frac{\alpha_{1} \alpha_{2} \cdots \alpha_{m} t^{m}}{\left(e_{\lambda}^{\alpha_{1}}(t)-1\right)\left(e_{\lambda}^{\alpha_{2}}(t)-1\right) \cdots\left(e_{\lambda}^{\alpha_{m}}(t)-1\right)} e^{z t} \\
=\sum_{n=0}^{\infty} B_{n, \lambda}^{(\alpha)}\left(z \mid \alpha_{1} \alpha_{2} \cdots \alpha_{m}\right) \frac{t^{n}}{n !}\end{array}$ & $g_{\lambda}(t)=\frac{\left(e_{\lambda}^{\alpha_{1}}(t)-1\right)\left(e_{\lambda}^{\alpha_{2}}(t)-1\right) \cdots\left(e_{\lambda}^{\alpha_{m}}(t)-1\right)}{\alpha_{1} \alpha_{2} \cdots \alpha_{m} t^{m}}$ \\
\hline $\begin{array}{l}\text { Degenerate generalized Euler } \\
\text { polynomials of order } m\end{array}$ & $\begin{array}{c}\frac{2^{m}}{\left(e_{\lambda}^{\alpha_{1}}(t)+1\right)\left(e_{\lambda}^{\alpha_{2}}(t)+1\right) \cdots\left(e_{\lambda}^{\alpha_{m}}(t)+1\right)} e^{z t} \\
\quad=\sum_{n=0}^{\infty} E_{n, \lambda}\left(z \mid \alpha_{1} \alpha_{2} \cdots \alpha_{m}\right) \frac{t^{n}}{n !}\end{array}$ & $g_{\lambda}(t)=\frac{\left(e_{\lambda}^{\alpha_{1}}(t)+1\right)\left(e_{\lambda}^{\alpha_{2}}(t)+1\right) \cdots\left(e_{\lambda}^{\alpha_{m}}(t)+1\right)}{2^{m}}$ \\
\hline $\begin{array}{c}\text { The degenerate new generalized } \\
\text { Bernoulli polynomials }\end{array}$ & $\frac{t^{m}}{e_{\lambda}(t)-\sum_{n=0}^{m-1} \frac{t^{n}}{n !}} e^{z t}=\sum_{n=0}^{\infty} B_{n, \lambda}^{[m-1]}(z) \frac{t^{n}}{n !}$ & $g_{\lambda}(t)=\frac{e_{\lambda}(t)-\sum_{n=0}^{m-1} t^{n}}{n !}$ \\
\hline
\end{tabular}

Table 1. List of some degenerate complex Appell polynomials.

The paper is organized as follows. In Section 2, we introduce degenerate complex Appell polynomials with cosine- and sine-Appell polynomials and prove some of their properties and relations. 
Section 3 presents illustrative examples of differential equations satisfied by degenerate complex Appell polynomials. Finally, Section 4 concludes the paper.

\section{Degenerate Complex Appell Polynomials}

In this section, we introduce degenerate complex Appell polynomials based on degenerate generating functions and study some of their properties. Furthermore, cosine- and sine-Appell polynomials are presented by splitting the complex Appell polynomials into their real $\Re$ and imaginary $\Im$ parts. We first provide the definition of degenerate Appell polynomials.

Definition 1. We define the sequence $\left\{A_{n, \lambda}(z)\right\}_{n=0}^{\infty}$ of degenerate complex Appell polynomials using the generating function

$$
\frac{1}{g_{\lambda}(t)} e^{z t}=\sum_{n=0}^{\infty} A_{n, \lambda}(z) \frac{t^{n}}{n !}, \quad A_{0}(z) \neq 0,
$$

where $a_{n, \lambda}:=A_{n, \lambda}(0)$ denotes the degenerate Appell numbers. Here, the degenerate generating functions $g_{\lambda}(t)$, which correspond to $g(t)$ in the Appell polynomials (1), are obtained by the degenerate exponential function $e_{\lambda}(t)$, which corresponds to the standard exponential function $e^{t}$ in $g(t)$ (see, for example, Table 1).

Remark 1. 1. Clearly, the first degenerate Appell number $a_{0, \lambda}$ is nonzero and is independent of $\lambda$ as $a_{0} \neq 0$ by definition (ii);

2. The sequence $\left\{A_{n, \lambda}(z)\right\}_{n=0}^{\infty}$ satisfies conditions (i) and (ii);

3. For $n \geq 0$, the sequence $\left\{A_{n, \lambda}(z)\right\}_{n=0}^{\infty}$ satisfies

$$
A_{n, \lambda}(z)=\sum_{k=0}^{n}\left(\begin{array}{l}
n \\
k
\end{array}\right) a_{n-k, \lambda} z^{k}
$$

as the identity for $A_{n, \lambda}(z)$ can be obtained by the binomial convolution of the sequences $\left\{a_{n, \lambda}\right\}_{n=0}^{\infty}$ and $\left\{z^{n}\right\}_{n=0}^{\infty}$.

On the basis of the degenerate complex Appell polynomials, we split complex values into real and imaginary parts using Euler's formula.

Definition 2. For a nonnegative integer $n$, we define the degenerate cosine-Appell polynomials $A_{n, \lambda}^{(c)}(x, y)$ and the degenerate sine-Appell polynomials $A_{n, \lambda}^{(s)}(x, y)$ by the following generating functions:

$$
\frac{1}{g_{\lambda}(t)} e^{x t} \cos (y t)=\sum_{n=0}^{\infty} A_{n, \lambda}^{(c)}(x, y) \frac{t^{n}}{n !}, \quad \frac{1}{g_{\lambda}(t)} e^{x t} \sin (y t)=\sum_{n=0}^{\infty} A_{n, \lambda}^{(s)}(x, y) \frac{t^{n}}{n !} .
$$

By Definitions 1 and 2, it follows that for $z=x+i y$,

$$
A_{n, \lambda}(z)=A_{n, \lambda}^{(c)}(x, y)+i A_{n, \lambda}^{(s)}(x, y)
$$

The degenerate trigonometric functions $A_{n, \lambda}^{(c)}(x, y)$ and $A_{n, \lambda}^{(s)}(x, y)$ have the following property.

Theorem 1. For $n \geq 0$ and $z=x+i y, A_{n, \lambda}^{(c)}(x, y)$ and $A_{n, \lambda}^{(s)}(x, y)$ in (3) can be represented in terms of $A_{n, \lambda}(z)$ and $A_{n, \lambda}(\bar{z})$ as follows:

$$
A_{n, \lambda}^{(c)}(x, y)=\frac{A_{n, \lambda}(z)+A_{n, \lambda}(\bar{z})}{2} \quad \text { and } \quad A_{n, \lambda}^{(s)}(x, y)=\frac{A_{n, \lambda}(z)-A_{n, \lambda}(\bar{z})}{2 i} .
$$

Proof. The proof follows easily by comparing the coefficients in the polynomial expansion in (1) and (2), and by using Euler's formula $e^{i y t}=\cos (y t)+i \sin (y t)$. 
Remark 2. It is noted that the sequences $\left\{A_{n, \lambda}^{(c)}(x, y)\right\}_{n=0}^{\infty}$ and $\left\{A_{n, \lambda}^{(s)}(x, y)\right\}_{n=0}^{\infty}$ can be explicitly determined when $g_{\lambda}(t)$ is given. For example, for $g_{\lambda}(t)=\frac{e_{\lambda}(t)+1}{2}$ and $g_{\lambda}(t)=\frac{e_{\lambda}(t)-1}{t}$ as defined in Table 1 , the first five polynomials can be listed as in Tables 2 and 3. One can verify that from [7], $\lim _{\lambda \rightarrow 0} E_{n, \lambda}^{(c)}(x, y)=E_{n}^{(c)}(x, y)$, $\lim _{\lambda \rightarrow 0} E_{n, \lambda}^{(s)}(x, y)=E_{n}^{(s)}(x, y), \lim _{\lambda \rightarrow 0} B_{n, \lambda}^{(c)}(x, y)=B_{n}^{(c)}(x, y)$, and $\lim _{\lambda \rightarrow 0} B_{n, \lambda}^{(s)}(x, y)=B_{n}^{(s)}(x, y)$. Moreover, Equation (4) holds.

We now provide an expression of $A_{n, \lambda}^{(c)}(x, y)$ and $A_{n, \lambda}^{(s)}(x, y)$ as well as some of their properties.

Theorem 2. Let $n$ be a non-negative integer. Then, the following identities hold:

$$
\begin{aligned}
& A_{n, \lambda}^{(c)}(x, y)=\sum_{m=0}^{\left[\frac{n}{2}\right]}\left(\begin{array}{c}
n \\
2 m
\end{array}\right)(-1)^{m} A_{n-2 m, \lambda}(x) y^{2 m}, \\
& A_{n, \lambda}^{(s)}(x, y)= \begin{cases}0, & \text { when } n=0, \\
\sum_{m=0}^{\left[\frac{n-1}{2}\right]}\left(\begin{array}{c}
n \\
2 m+1
\end{array}\right)(-1)^{m} A_{n-2 m-1, \lambda}(x) y^{2 m+1}, & \text { when } n \in \mathbb{N},\end{cases}
\end{aligned}
$$

where $[n]$ represents the greatest integer less than or equal to $n$.

Proof. By considering the product of $\frac{1}{g_{\lambda}(t)} e^{x t}$ and $\cos (y t)$, the identity for $A_{n, \lambda}^{(c)}(x, y)$ is easily obtained by the binomial convolution of the sequences $\left\{A_{n, \lambda}(x)\right\}_{n=0}^{\infty}$ and $\left\{\frac{1+(-1)^{n}}{2} i^{n} y^{n}\right\}_{n=0}^{\infty}$. Similarly, the identity for $A_{n, \lambda}^{(s)}(x, y)$ can be obtained by the binomial convolution of the sequences $\left\{A_{n, \lambda}(x)\right\}_{n=0}^{\infty}$ and $\left\{\frac{1-(-1)^{n}}{2 i} i^{n} y^{n}\right\}_{n=0}^{\infty}$.

Theorem 3. If we let $g_{\lambda}(t)=\sum_{n=0}^{\infty} \hat{a}_{n, \lambda} \frac{t^{n}}{n !}$ for some sequence $\left\{\hat{a}_{n, \lambda}\right\}_{n=0}^{\infty}$, then the followings hold:

$$
\begin{aligned}
& \sum_{k=0}^{n}\left(\begin{array}{l}
n \\
k
\end{array}\right) \hat{a}_{n-k, \lambda} A_{k, \lambda}^{(c)}(x, y)=\sum_{k=0}^{\left[\frac{n}{2}\right]}\left(\begin{array}{c}
n \\
2 k
\end{array}\right)(-1)^{k} x^{n-2 k} y^{2 k}, \\
& \sum_{k=0}^{n}\left(\begin{array}{l}
n \\
k
\end{array}\right) \hat{a}_{n-k, \lambda} A_{k, \lambda}^{(s)}(x, y)=\sum_{k=0}^{\left[\frac{n-1}{2}\right]}\left(\begin{array}{c}
n \\
2 k+1
\end{array}\right)(-1)^{m} x^{n-2 k-1} y^{2 k+1} .
\end{aligned}
$$

Proof. We only prove the first formula, as the proof of the second is similar. We consider the product $e^{x t} \cos (y t)$. First, using the binomial convolution of the sequences $\left\{x^{n}\right\}_{n=0}^{\infty}$ and $\left\{\frac{1+(-1)^{n}}{2} i^{n} y^{n}\right\}_{n=0}^{\infty}$, we have that $e^{x t} \cos (y t)$ is the exponential generating function of the sequence

$$
\left\{\sum_{k=0}^{\left[\frac{n}{2}\right]}\left(\begin{array}{c}
n \\
2 k
\end{array}\right)(-1)^{k} x^{n-2 k} y^{2 k}\right\}_{n=0}^{\infty} .
$$

Then, by rewriting the product $e^{x}(t) \cos (y t)$ as

$$
g_{\lambda}(t)\left(\frac{1}{g_{\lambda}(t)} e^{x t} \cos (y t)\right)
$$

and using the binomial convolution of the sequences $\left\{\hat{a}_{n, \lambda}\right\}_{n=0}^{\infty}$ and $\left\{A_{n, \lambda}(z)\right\}_{n=0}^{\infty}$, we obtain that $e^{x t} \cos (y t)$ is the exponential generating function of the sequence

$$
\left\{\sum_{k=0}^{n}\left(\begin{array}{l}
n \\
k
\end{array}\right) \hat{a}_{n-k, \lambda} A_{k, \lambda}^{(c)}(x, y)\right\}_{n=0}^{\infty} .
$$


Thus, the first identity is proved.

Before providing an example of Theorem 3 , we note that the $\lambda$-falling factorial sequence $(x)_{n, \lambda}$ is given by the degenerate exponential function as follows $[10,15]$ :

$$
e_{\lambda}^{x}(t):=(1+\lambda t)^{\frac{x}{\lambda}}=\sum_{n=0}^{\infty}(x)_{n, \lambda} \frac{t^{n}}{n !}
$$

where

$$
(x)_{0, \lambda}=1, \quad(x)_{n, \lambda}=x(x-\lambda)(x-2 \lambda) \cdots(x-(n-1) \lambda) \quad(n \geq 1) .
$$

Example 1. Type 2 degenerate Euler polynomials $E_{n, \lambda}^{(2)}(z)$ are defined by the generating functions

$$
\frac{2}{e_{\lambda}^{\frac{1}{2}}(t)+e_{\lambda}^{-\frac{1}{2}}(t)} e^{z t}=\sum_{n=0}^{\infty} E_{n, \lambda}^{(2)}(z) \frac{t^{n}}{n !} .
$$

One can verify that the identities in Theorem 3 hold because $\hat{a}_{n, \lambda}=\frac{1}{2}\left(\left(\frac{1}{2}\right)_{n, \lambda}+\left(-\frac{1}{2}\right)_{n, \lambda}\right)$.

We now show that the degenerate cosine- and sine-Appell polynomials $\left(A_{n, \lambda}^{(c)}(x, y)\right.$ and $\left.A_{n, \lambda}^{(s)}(x, y)\right)$ are represented by the Stirling numbers of the second kind $\mathcal{S}_{2}(n, m)$.

Theorem 4. For $n \in \mathbb{N} \cup\{0\}$, the degenerate cosine-and sine-Appell polynomials satisfy

$$
A_{n, \lambda}^{(c)}(x, y)=\sum_{k=0}^{n}\left(\begin{array}{l}
n \\
k
\end{array}\right) x^{k} A_{n-k, \lambda}^{(c)}(0, y) \quad \text { and } \quad A_{n, \lambda}^{(s)}(x, y)=\sum_{k=0}^{n}\left(\begin{array}{l}
n \\
k
\end{array}\right) x^{k} A_{n-k, \lambda}^{(s)}(0, y)
$$

Proof. We first note that $e^{x t}$ is the exponential generating function of the sequence $\left\{x^{n}\right\}_{n=0}^{\infty}$. Then, considering the product $\frac{1}{g_{\lambda}(t)} \cos (y t)$ and $e^{x t}$, the binomial convolution of the sequences $\left\{A_{n, \lambda}^{(c)}(0, y)\right\}_{n=0}^{\infty}$ and $\left\{x^{n}\right\}_{n=0}^{\infty}$ implies that $\frac{1}{g_{\lambda}(t)} e^{x t} \cos (y t)$ is the exponential generating function of the sequence

$$
\left\{\sum_{k=0}^{n}\left(\begin{array}{l}
n \\
k
\end{array}\right) x^{k} A_{n-k, \lambda}^{(c)}(0, y)\right\}_{n=0}^{\infty} .
$$

Similarly, by the binomial convolution of the sequences $\left\{A_{n, \lambda}^{(s)}(0, y)\right\}_{n=0}^{\infty}$ and $\left\{x^{n}\right\}_{n=0}^{\infty}$, we have that $\frac{1}{g_{\lambda}(t)} e^{x t} \sin (y t)$ is the exponential generating function of the sequence

$$
\left\{A_{n, \lambda}^{(s)}(x, y)=\sum_{k=0}^{n}\left(\begin{array}{l}
n \\
k
\end{array}\right) x^{k} A_{n-k, \lambda}^{(s)}(0, y)\right\}_{n=0}^{\infty} .
$$

Recalling that $x^{n}=\sum_{k=0}^{n} \mathcal{S}_{2}(n, k)(x)_{k}$ (see [3,16]), where $\mathcal{S}_{2}(n, k)$ and $(x)_{k}$ are the Stirling numbers of the second kind and the falling factorial, respectively, we have the following corollary.

Corollary 1. For $n \in \mathbb{N} \cup\{0\}$, the following identities hold:

$$
\begin{aligned}
& A_{n, \lambda}^{(c)}(x, y)=\sum_{k=0}^{n} \sum_{m=0}^{k}\left(\begin{array}{l}
n \\
k
\end{array}\right) \mathcal{S}_{2}(k, m)(x)_{m} A_{n-k, \lambda}^{(c)}(0, y), \\
& A_{n, \lambda}^{(s)}(x, y)=\sum_{k=0}^{n} \sum_{m=0}^{k}\left(\begin{array}{l}
n \\
k
\end{array}\right) \mathcal{S}_{2}(k, m)(x)_{m} A_{n-k, \lambda}^{(s)}(0, y) .
\end{aligned}
$$


Table 2. Expressions of the first five $E_{n, \lambda}^{(c)}(x, y), E_{n, \lambda}^{(s)}(x, y)$, and $E_{n, \lambda}(z)$.

\begin{tabular}{|c|c|c|c|}
\hline$n$ & $E_{n, \lambda}^{(c)}(x, y)$ & $E_{n, \lambda}^{(s)}(x, y)$ & $E_{n, \lambda}(z), z=x+i y$ \\
\hline 0 & 1 & 0 & 1 \\
\hline 1 & $x-\frac{1}{2}$ & $y$ & $z-\frac{1}{2}$ \\
\hline 2 & $\frac{1}{2}\left(\lambda+2 x^{2}-2 x-2 y^{2}\right)$ & $\frac{1}{2}\left(x-\frac{1}{2}\right)$ & $\frac{1}{2}(\lambda+2 z(z-1))$ \\
\hline 3 & $-\lambda^{2}+\frac{3}{2} \lambda x+\frac{1}{4}(2 x-1)\left(2 x^{2}-2 x-6 y^{2}-1\right)$ & $\frac{1}{2}\left(3 \lambda+6 x^{2}-6 x-2 y^{2}\right)$ & $\frac{1}{4}\left(-4 \lambda^{2}+6 \lambda z+4 z^{3}-6 z^{2}+1\right)$ \\
\hline 4 & $3 \lambda^{3}-4 \lambda^{2} x+\frac{\lambda}{2}\left(6 x^{2}-6 y^{2}-3\right)+\left(x^{4}-2 x^{3}-6 x^{2} y^{2}+6 x y^{2}+x+y^{4}\right)$ & $y\left(-4 \lambda^{2}+6 \lambda x+4 x^{3}-6 x^{2}-4 x y^{2}+2 y^{2}+1\right)$ & $\left(3 \lambda^{3}-4 \lambda^{2} z+\frac{\lambda}{2}\left(6 z^{2}-3\right)+\left(z^{4}-2 z^{3}+z\right)\right)$ \\
\hline
\end{tabular}

Table 3. Expressions of the first five $B_{n, \lambda}^{(c)}(x, y), B_{n, \lambda}^{(s)}(x, y)$, and $B_{n, \lambda}(z)$.

\begin{tabular}{lccc}
\hline$n$ & $\boldsymbol{B}_{n, \lambda}^{(c)}(x, y)$ & $\boldsymbol{B}_{n, \lambda}^{(s)}(x, y)$ & $\boldsymbol{B}_{n, \lambda}(z), z=x+i y$ \\
\hline 0 & 1 & 0 & 1 \\
1 & $\frac{\lambda-1}{2}+x$ & $y$ & $\frac{\lambda-1}{2}+z$ \\
2 & $\frac{1}{6}\left(-\lambda^{2}+6 \lambda x+6 x^{2}-6 x-6 y^{2}+1\right)$ & $y(\lambda+2 x-1)$ & $\frac{1}{6}\left(-\lambda^{2}+6 \lambda z+6 z^{2}-6 z+1\right)$ \\
3 & $\frac{1}{2}\left(\lambda^{3}-2 \lambda^{2} x+\lambda\left(6 x^{2}-6 y^{2}-1\right)+2(2 x-1)\left(x^{2}-x-3 y^{2}\right)\right)$ & $-\frac{1}{2} y\left(\lambda^{2}-6 \lambda x-6 x^{2}+6 x+2 y^{2}-1\right)$ & $\frac{1}{2}\left(\lambda^{3}-2 \lambda^{2} z+\lambda\left(6 z^{2}-1\right)+2 z\left(2 z^{2}-3 z+1\right)\right)$ \\
4 & $-\frac{19}{30} \lambda^{4}+\lambda^{3} x+\lambda^{2}\left(-x^{2}+y^{2}+\frac{2}{3}\right)+\lambda x\left(2 x^{2}-6 y^{2}-1\right)+$ & $\frac{1}{2} y \lambda^{3}-\lambda^{2} x y+\lambda\left(3 x^{2}-y^{2}-\frac{1}{2}\right) y+$ & $-\frac{19}{30} \lambda^{4}+\lambda^{3} z+\lambda^{2}\left(\frac{2}{3}-z^{2}\right)+$ \\
& $x^{4}-2 x^{3}-x^{2}\left(6 y^{2}-1\right)+6 x y^{2}+y^{4}-y^{2}-\frac{1}{30}$ & $y(2 x-1)\left(x^{2}-x-y^{2}\right)$ & $\lambda z\left(2 z^{2}-1\right)+z^{4}-2 z^{3}+z^{2}-\frac{1}{30}$ \\
\hline
\end{tabular}


Example 2. 1. If $g_{\lambda}(t)=\frac{e_{\lambda}(t)+1}{2}$, then we have the sequence $\left\{E_{n, \lambda}(z)\right\}_{n=0}^{\infty}$ of the degenerate complex Euler polynomials in Table 1. Thus, the degenerate cosine- and sine-Euler polynomials can be obtained by

$$
\begin{aligned}
& E_{n, \lambda}^{(c)}(x, y)=\sum_{k=0}^{n} \sum_{m=0}^{k}\left(\begin{array}{l}
n \\
k
\end{array}\right) \mathcal{S}_{2}(k, m)(x)_{m} E_{n-k, \lambda}^{(c)}(0, y), \\
& E_{n, \lambda}^{(s)}(x, y)=\sum_{k=0}^{n} \sum_{m=0}^{k}\left(\begin{array}{l}
n \\
k
\end{array}\right) \mathcal{S}_{2}(k, m)(x)_{m} E_{n-k, \lambda}^{(s)}(0, y) ;
\end{aligned}
$$

2. When $g_{\lambda}(t)=\frac{e_{\lambda}(t)-1}{t}$, we obtain the sequence $\left\{B_{n, \lambda}(z)\right\}_{n=0}^{\infty}$ of the degenerate complex Bernoulli polynomials in Table 1. Hence, the degenerate cosine- and sine-Bernoulli polynomials are given by

$$
\begin{aligned}
& B_{n, \lambda}^{(c)}(x, y)=\sum_{k=0}^{n} \sum_{m=0}^{k}\left(\begin{array}{l}
n \\
k
\end{array}\right) \mathcal{S}_{2}(k, m)(x)_{m} B_{n-k, \lambda}^{(c)}(0, y), \\
& B_{n, \lambda}^{(s)}(x, y)=\sum_{k=0}^{n} \sum_{m=0}^{k}\left(\begin{array}{l}
n \\
k
\end{array}\right) \mathcal{S}_{2}(k, m)(x)_{m} B_{n-k, \lambda}^{(s)}(0, y) .
\end{aligned}
$$

Remark 3. In particular, when $g_{\lambda}(t)=1$ in Definition 1 , we consider the cosine-and sine- sequences $C_{n}(x, y)$ and $S_{n}(x, y)$ given by (see [8])

$$
e^{x t} \cos (y t)=\sum_{n=0}^{\infty} C_{n}(x, y) \frac{t^{n}}{n !} \text { and } e^{x t} \sin (y t)=\sum_{n=0}^{\infty} S_{n}(x, y) \frac{t^{n}}{n !} .
$$

Then, as $A_{n, \lambda}(x)=x^{n}$ for $g_{\lambda}(t)=1$ in Theorem 2, we have that

$$
\begin{aligned}
& C_{n}(x, y)=\sum_{m=0}^{\left[\frac{n}{2}\right]}\left(\begin{array}{c}
n \\
2 m
\end{array}\right)(-1)^{m} y^{2 m} x^{n-2 m}, \\
& S_{n}(x, y)=\sum_{m=0}^{\left[\frac{n-1}{2}\right]}\left(\begin{array}{c}
n \\
2 m+1
\end{array}\right)(-1)^{m} y^{2 m+1} x^{n-2 m-1} .
\end{aligned}
$$

The following two theorems show that the degenerate complex Appell polynomials can be split into $C_{n}(x, y)$ and $S_{n}(x, y)$.

Theorem 5. For $n \in \mathbb{N} \cup\{0\}$, the degenerate complex Appell polynomials are related to $C_{n}(x, y)$ and $S_{n}(x, y)$ as follows:

$$
A_{n, \lambda}(z)=\sum_{k=0}^{n}\left(\begin{array}{l}
n \\
k
\end{array}\right) a_{n-k, \lambda}\left(C_{k}(x, y)+i S_{k}(x, y)\right), \quad z:=x+i y \in \mathbb{C} .
$$

Proof. As $\frac{1}{g_{\lambda}(t)} e^{x t}(\cos (y t)+i \sin (y t))$ is the exponential generating functions for $A_{n, \lambda}(z)$, we immediately have (5) by the binomial convolution of the sequences $\left\{a_{n, \lambda}\right\}_{n=0}^{\infty}$ and $\left\{C_{n}(x, y)+\right.$ $\left.i S_{n}(x, y)\right\}_{n=0}^{\infty}$, as $\frac{1}{g_{\lambda}(t)}=\sum_{n=0}^{\infty} a_{n, \lambda} \frac{t^{n}}{n !}$.

Theorem 6. For $r, s \geq 0$, the degenerate cosine- and sine-Appell polynomials have the following properties:

$$
\begin{aligned}
& A_{n, \lambda}^{(c)}(x \pm r, y \pm s)=\sum_{l=0}^{n}\left(\begin{array}{l}
n \\
l
\end{array}\right)( \pm 1)^{n-l}\left[A_{l, \lambda}^{(c)}(x, y) C_{n-l}(r, s)-A_{l, \lambda}^{(s)}(x, y) S_{n-l}(r, s)\right] \\
& A_{n}^{(s)}(x \pm r, y \pm s)=\sum_{l=0}^{n}\left(\begin{array}{l}
n \\
l
\end{array}\right)( \pm 1)^{n-l}\left[A_{l, \lambda}^{(s)}(x, y) C_{n-l}(r, s)-A_{l, \lambda}^{(c)}(x, y) S_{n-l}(r, s)\right] .
\end{aligned}
$$


Proof. Let us consider the trigonometric identities

$$
\begin{aligned}
& e^{(x \pm r) t} \cos ((y \pm s) t)=e^{x t} \cos (y t) e^{ \pm r t} \cos ( \pm s t)-e^{x t} \sin (y t) e^{ \pm r t} \sin ( \pm s t), \\
& e^{(x \pm r) t} \sin ((y \pm s) t)=e^{x t} \sin (y t) e^{ \pm r t} \cos ( \pm s t)+e^{x t} \cos (y t) e^{ \pm r t} \sin ( \pm s t) .
\end{aligned}
$$

We note that $C_{n}( \pm x, \pm y)=( \pm 1)^{n} C_{n}(x, y)$ and $S_{n}( \pm x, \pm y)=( \pm 1)^{n} S_{n}(x, y)$ in Remark 3. As $\frac{1}{g_{\lambda}(t)} e^{(x \pm r) t} \cos ((y \pm s) t)$ is the exponential generating function for $A_{n, \lambda}^{(c)}(x \pm r, y \pm s)$, we obtain (6) by the binomial convolutions. Equation (7) can be proved similarly.

Theorem 7. For $r>0$, the following identities for degenerate cosine- and sine-Appell polynomials hold:

$$
\begin{aligned}
& A_{n, \lambda}^{(c)}(r \pm x, y)=\sum_{k=0}^{n}\left(\begin{array}{l}
n \\
k
\end{array}\right) A_{k, \lambda}(r)( \pm 1)^{n-k} C_{n-k}(x, y), \\
& A_{n}^{(s)}(r \pm x, y)=\sum_{k=0}^{n}\left(\begin{array}{l}
n \\
k
\end{array}\right) A_{k, \lambda}(r)( \pm 1)^{n-k} S_{n-k}(x, y) .
\end{aligned}
$$

Proof. As $\frac{1}{g_{\lambda}(t)} e^{r t} e^{ \pm x t} \cos ( \pm y t)$ is the exponential generating function for $A_{n}^{(c)}(r \pm x, y)$, we obtain (8) by the binomial convolution of the sequences $\left\{A_{n, \lambda}(r)\right\}_{n=0}^{\infty}$ and $\left\{C_{n}(x, y)( \pm 1)^{n}\right\}_{n=0}^{\infty}$. Similarly, (9) can be proved.

As $A_{n, \lambda}(r)$ is obtained by the binomial convolution of the sequences $\left\{a_{n, \lambda}\right\}_{n=0}^{\infty}$ and $\left\{r^{n}\right\}_{n=0}^{\infty}$, we obtain the following corollary.

Corollary 2. For $r>0$, the following identities for $A_{n, \lambda}^{(c)}(x, y)$ and $A_{n, \lambda}^{(s)}(x, y)$ hold:

$$
\begin{aligned}
& A_{n, \lambda}^{(c)}(r \pm x, y)=\sum_{k=0}^{n}\left(\begin{array}{l}
n \\
k
\end{array}\right)\left(\sum_{j=0}^{k}\left(\begin{array}{l}
k \\
j
\end{array}\right) a_{j, \lambda} r^{k-j}\right)( \pm 1)^{n-k} C_{n-k}(x, y), \\
& A_{n}^{(s)}(r \pm x, y)=\sum_{k=0}^{n}\left(\begin{array}{l}
n \\
k
\end{array}\right)\left(\sum_{j=0}^{k}\left(\begin{array}{l}
k \\
j
\end{array}\right) a_{j, \lambda} r^{k-j}\right)( \pm 1)^{n-k} S_{n-k}(x, y) .
\end{aligned}
$$

\section{Illustrative Examples of Differential Equations}

In this section, we present two examples of degenerate complex Appell polynomials: the degenerate Bernoulli and Euler polynomials. By using quasi-monomiality, we derive the related differential equation satisfied by each of these polynomials. To this end, we first recall the definition of quasi-monomiality and some related properties.

By $[3,17]$, a polynomial set $\left\{p_{n}(z)\right\}_{n=0}^{\infty}$ is called quasi-monomial if there exist two linear operators $\widehat{P}$ and $\widehat{M}$ such that

$$
\widehat{P}\left(p_{n}(z)\right)=n p_{n-1}(z) \text { and } \widehat{M}\left(p_{n}(z)\right)=p_{n+1}(z), \quad n \in \mathbb{N},
$$

where $\widehat{P}$ and $\widehat{M}$ are called the derivative and multiplication operators, respectively.

On the basis of the results related to the monomiality principle, the quasi-monomial polynomials $\left\{p_{n}\right\}$ satisfy the following differential equation:

$$
\widehat{M} \widehat{P} p_{n}(z)=n p_{n}(z)
$$

In particular, as the sequence $\left\{A_{n, \lambda}(z)\right\}_{n=0}^{\infty}$ is Appell-type, we have (more details are in [3]): 


$$
\widehat{P}=D_{z} \quad \text { and } \quad \widehat{M}=-\frac{g_{\lambda}^{\prime}\left(D_{z}\right)}{g_{\lambda}\left(D_{z}\right)}+z, \quad D_{z}=\frac{d}{d z}
$$

or equivalently,

$$
\left(-\frac{g_{\lambda}^{\prime}\left(D_{z}\right)}{g_{\lambda}\left(D_{z}\right)} D_{z}+z D_{z}\right) A_{n, \lambda}(z)=n A_{n, \lambda}(z)
$$

3.1. Degenerate complex Bernoulli polynomials $B_{n, \lambda}(z)$

The degenerate complex Bernoulli polynomials $B_{n, \lambda}(z)$ are defined by the degenerate generating function $g_{\lambda}(t)=\frac{e_{\lambda}(t)-1}{t}$. That is,

$$
\begin{aligned}
& \frac{1}{g_{\lambda}(t)}=\frac{t}{e_{\lambda}(t)-1}=\sum_{n=0}^{\infty} b_{n, \lambda} \frac{t^{n}}{n !}, \\
& \frac{t}{e_{\lambda}(t)-1} e^{z t}=\sum_{n=0}^{\infty} B_{n, \lambda}(z) \frac{t^{n}}{n !}=\sum_{n=0}^{\infty}\left(\sum_{k=0}^{n}\left(\begin{array}{l}
n \\
k
\end{array}\right) b_{n-k, \lambda} z^{k}\right) \frac{t^{n}}{n !} .
\end{aligned}
$$

By simple computations, it is easily seen that the following expansion holds true:

$$
-t \frac{g_{\lambda}^{\prime}(t)}{g_{\lambda}(t)}=\frac{-t e_{\lambda}(t)+(1+\lambda t)\left(e_{\lambda}(t)-1\right)}{(1+\lambda t)\left(e_{\lambda}(t)-1\right)}=\tilde{b}_{0, \lambda} t+\tilde{b}_{1, \lambda} \frac{t^{2}}{1 !}+\tilde{b}_{2, \lambda} \frac{t^{3}}{2 !}+\tilde{b}_{3, \lambda} \frac{t^{4}}{3 !}+\cdots,
$$

where the first six coefficients are the following:

$$
\begin{array}{ll}
\tilde{b}_{0, \lambda}=\frac{\lambda-1}{2}, & \tilde{b}_{1, \lambda}=\frac{1}{12}\left(-5 \lambda^{2}+6 \lambda-1\right), \\
\tilde{b}_{2, \lambda}=\frac{\lambda}{4}\left(3 \lambda^{2}-4 \lambda+1\right), & \tilde{b}_{3, \lambda}=\frac{1}{120}\left(-251 \lambda^{4}+360 \lambda^{3}-110 \lambda^{2}+1\right), \\
\tilde{b}_{4, \lambda}=\frac{1}{12} \lambda\left(95 \lambda^{4}-144 \lambda^{3}+50 \lambda^{2}-1\right), & \\
\tilde{b}_{5, \lambda}=\frac{1}{504}\left(-19087 \lambda^{6}+30240 \lambda^{5}-11508 \lambda^{4}+357 \lambda^{2}-2\right) . &
\end{array}
$$

As the operators for the degenerate complex Bernoulli polynomials $B_{n, \lambda}(z)$ are given by

$$
\widehat{P}=D_{z} \quad \text { and } \quad \widehat{M}=\frac{-D_{z} e_{\lambda}\left(D_{z}\right)+\left(1+\lambda D_{z}\right)\left(e_{\lambda}\left(D_{z}\right)-1\right)}{D_{z}\left(1+\lambda D_{z}\right)\left(e_{\lambda}\left(D_{z}\right)-1\right)}+z
$$

the degenerate complex Bernoulli polynomials satisfy the following differential equation:

$$
\left(\frac{-D_{z} e_{\lambda}\left(D_{z}\right)+\left(1+\lambda D_{z}\right)\left(e_{\lambda}\left(D_{z}\right)-1\right)}{\left(1+\lambda D_{z}\right)\left(e_{\lambda}\left(D_{z}\right)-1\right)}+z D_{z}\right) B_{n, \lambda}(z)=n B_{n, \lambda}(z), \quad e_{\lambda}\left(D_{z}\right)=\left(1+\lambda D_{z}\right)^{\frac{1}{\lambda}} .
$$

We note that for the degenerate complex Bernoulli polynomials $B_{n, \lambda}(z)$ of degree $n$, the differential equation is equivalently expressed by

$$
\left(\sum_{k=0}^{n-1} \frac{\tilde{b}_{k, \lambda}}{k !} D_{z}^{k+1}+z D_{z}\right) B_{n, \lambda}(z)=n B_{n, \lambda}(z) .
$$

\subsection{Degenerate Complex Euler Polynomials $E_{n, \lambda}(z)$}

The degenerate complex Euler polynomials $E_{n, \lambda}(z)$ are given by the degenerate generating function $g_{\lambda}(t)=\frac{e_{\lambda}(t)+1}{2}$. Equivalently, 


$$
\begin{aligned}
& \frac{1}{g_{\lambda}(t)}=\frac{2}{e_{\lambda}(t)+1}=\sum_{n=0}^{\infty} e_{n, \lambda} \frac{t^{n}}{n !} \\
& \frac{2}{e_{\lambda}(t)+1} e^{z t}=\sum_{n=0}^{\infty} E_{n, \lambda}(z) \frac{t^{n}}{n !}=\sum_{n=0}^{\infty}\left(\sum_{k=0}^{n}\left(\begin{array}{l}
n \\
k
\end{array}\right) e_{n-k, \lambda} z^{k}\right) \frac{t^{n}}{n !} .
\end{aligned}
$$

The expansion for $-\frac{g_{\lambda}^{\prime}(t)}{g_{\lambda}(t)}$ is given by

$$
-\frac{g_{\lambda}^{\prime}(t)}{g_{\lambda}(t)}=\frac{-e_{\lambda}(t)}{(1+\lambda t)\left(e_{\lambda}(t)+1\right)}=\tilde{e}_{0, \lambda}+\tilde{e}_{1, \lambda} \frac{t}{1 !}+\tilde{e}_{2, \lambda} \frac{t^{2}}{2 !}+\tilde{e}_{3, \lambda} \frac{t^{3}}{3 !}+\cdots,
$$

where the first six coefficients are the following:

$$
\begin{array}{rlrl}
\tilde{e}_{0, \lambda} & =-\frac{1}{2}, & \tilde{e}_{1, \lambda}=\frac{1}{4}(\lambda-1), \\
\tilde{e}_{2, \lambda}=\frac{1}{4} \lambda(\lambda-3), & \tilde{e}_{3, \lambda}=\frac{1}{8}\left(3 \lambda^{3}-19 \lambda^{2}+3 \lambda+1\right), \\
\tilde{e}_{4, \lambda}=-\frac{1}{4} \lambda\left(3 \lambda^{3}-35 \lambda^{2}+15 \lambda+5\right), & \\
\tilde{e}_{5, \lambda}=\frac{1}{8}\left(15 \lambda^{5}-303 \lambda^{4}+235 \lambda^{3}+65 \lambda^{2}-10 \lambda-2\right) . &
\end{array}
$$

As the operators for the degenerate complex Euler polynomials $E_{n, \lambda}(z)$ are given by

$$
\widehat{P}=D_{z} \quad \text { and } \quad \widehat{M}=\frac{-e_{\lambda}\left(D_{z}\right)}{\left(1+\lambda D_{z}\right)\left(e_{\lambda}\left(D_{z}\right)+1\right)}+z,
$$

the differential equation of the degenerate complex Euler polynomials is

$$
\left(\frac{-e_{\lambda}\left(D_{z}\right)}{\left(1+\lambda D_{z}\right)\left(e_{\lambda}\left(D_{z}\right)+1\right)} D_{z}+z D_{z}\right) E_{n, \lambda}(z)=n E_{n, \lambda}(z) .
$$

It is noted that for the degenerate complex Euler polynomials $E_{n, \lambda}(z)$ of degree $n$, the differential equation is equivalently given by

$$
\left(\sum_{k=0}^{n-1} \frac{\tilde{e}_{k, \lambda}}{k !} D_{z}^{k+1}+z D_{z}\right) E_{n, \lambda}(z)=n E_{n, \lambda}(z)
$$

\section{Conclusions}

We studied general properties and related identities of degenerate complex Appell polynomials obtained by degenerate versions of the generating functions of complex Appell polynomials. Treating the real and imaginary parts separately, we introduced the degenerate cosine-Appell and sine-Appell polynomials and presented some of their properties and relations, as well as examples. These polynomials are expressed in terms of the Stirling numbers of the second kind, and several identities were proved in terms of the cosine- and sine- polynomial sequences $C_{n}(x, y)$ and $S_{n}(x, y)$ generated by $e^{x t} \cos (y t)$ and $e^{x t} \sin (y t)$ in Remark 3. In addition, on the basis of quasi-monomiality, the differential equations satisfied by degenerate complex Bernoulli and Euler polynomials were derived.

Author Contributions: Conceptualization, D.K.; methodology, D.K.; validation, D.K. and S.K.; formal analysis, D.K. and S.K.; investigation, S.K.; resources, S.K. and D.K.; writing-original draft preparation, D.K.; writing-review and editing, S.K.; visualization, S.K.; supervision, S.K.; project administration, S.K.; funding acquisition, S.K.

Funding: This work was supported by the National Research Foundation of Korea (NRF) Grant funded by the Korean Government (MSIP) (NRF-2017R1A5A1015722) and Pusan National University Research Grant, 2018. 
Acknowledgments: We would like to thank the referees for their comments and suggestions which greatly improved the original manuscript.

Conflicts of Interest: The authors declare no conflict of interest.

\section{References}

1. Boas, R.P.; Buck, R.C. Polynomial Expansions of Analytic Functions; Springer \& Acad. Press: Berlin/Heidelberg, Germany, 1958.

2. Bateman, H.; Erdélyi, A. (Eds.) Higher Transcendental Functions; McGraw-Hill: New York, NY, USA, 1953-1955; Volume 1-3.

3. Khan, S.; Raza, N. General-Appell Polynomials within the Context of Monomiality Principle. Int. J. Anal. 2013, 2013, 328032. [CrossRef]

4. Kim, T.; Kim, D.S.; Jang, L.-C.; Kim, H.-Y. On type 2 degenerate Bernoulli and Euler polynomials of complex variable. Adv. Differ. Equ. 2019, 2019, 490. [CrossRef]

5. Appell, P.E. Sur une classe de polynômes. Ann. Sci. École Norm. Sup. 1880, 9, 119-144. [CrossRef]

6. Wood, B. Generalized Szász operators for the approximation in the complex domain. SIAM J. Appl. Math. 1969, 17, 790-801. [CrossRef]

7. Kim, D. A Note on the Degenerate Type of Complex Appell Polynomials. Symmetry 2019, 11, 1339. [CrossRef]

8. Kim, D.S.; Kim, T.; Lee, H. A note on degenerate Euler and Bernoulli polynomials of complex variable. Symmetry 2019, 11, 1168. [CrossRef]

9. Kim, T.; Kim, D.S. Degenerate central Bell numbers and polynomials. Rev. R. Acad. Clenc. Exactas Fis. Nat. Ser. A Mat. RACSAM 2019, 113, 2507-2513. [CrossRef]

10. Kim, T.; Kim, D.S. Degenerate Laplace transform and degenerate gamma function. Russ. J. Math. Phys. 2017, 24, 241-248. [CrossRef]

11. Pyo, S.-S. Degenerate Cauchy numbers and polynomials of the fourth kind. Adv. Stud. Contemp. Math. (Kyungshang) 2018, 28, 127-138.

12. Carlitz, L. Degenerate Stirling, Bernoulli and Eulerian numbers. Utilitas Math. 1979, 15, 51-88.

13. Carlitz, L.; Riordan, J. The divided central differences of zero. Can. J. Math. 1963, 15, 94-100. [CrossRef]

14. Kim, D.S.; Kim, T.; Jang, G.-W. A note on degenerate Stirling numbers of the first kind. Proc. Jangjeon Math. Soc. 2018, 21, 393-404.

15. Kim, T.; Jang, G.-W. A note on degenerate gamma function and degenerate Stirling number of the second kind. Adv. Stud. Contemp. Math. (Kyungshang) 2018, 28, 207-214.

16. Kim, T.; Yao, Y.; Kim, D.S.; Jang, G.-W. Degenerate $r$-Stirling numbers and $r$-Bell polynomials. Russ. J. Math. Phys. 2018, 25, 44-58. [CrossRef]

17. Srivastava, H.M.; Ricci, P.E.; Natalini, P. A family of complex Appell polynomial sets. Rev. de la Real Acad. de Cienc. Exactas Físicas y Nat. Ser. A. Mat. 2019, 113, 2359-2371. [CrossRef] 